\title{
Artificial Bee Colony Optimization to Reallocate Personnel to Tasks Improving Workplace Safety
}

\author{
Beatrice Lazzerini and Francesco Pistolesi \\ Department of Information Engineering, University of Pisa \\ Largo Lucio Lazzarino 1, 56122 Pisa, Italy \\ \{b.lazzerini,f.pistolesi\}@iet.unipi.it
}

\begin{abstract}
Worldwide, just under 5,800 people go to work every day and do not return because they die on the job. The groundbreaking Industry 4.0 paradigm includes innovative approaches to improve the safety in the workplace, but Small and Medium Enterprises (SMEs) - which represent $99 \%$ of the companies in the $\mathrm{EU}$ - are often unprepared to the high costs for safety. A cost-effective way to improve the level of safety in SMEs may be just to reassign employees to tasks, so as to assign hazardous tasks to the more cautious employees. This paper presents a multi-objective approach to reallocate the personnel of a company to the tasks in order to maximize the workplace safety, while minimizing the cost, and the time to learn the new tasks assigned. Pareto-optimal reallocations are first generated using the Non-dominated Sorting artificial Bee Colony (NSBC) algorithm, and the best one is then selected using the Technique for Order of Preference by Similarity to Ideal Solution (TOPSIS). The approach was tested in two SMEs with 11 and 25 employees, respectively.
\end{abstract}

Keywords: Bee colony algorithm, Occupational safety and health, Multiobjective optimization, Personnel reallocation, Risk perception, TOPSIS.

\section{Introduction}

Improving the safety of work environments is key. Every 15 seconds a worker dies as a consequence of occupational injuries and accidents [1]. New technologies helps save a countless number of lives today, but workplace fatalities are not keeping on diminishing enough.

In economic terms, occupational illnesses and accidents at work result in costs up to $6 \%$ of GDP, in country estimates [1]. Statistics also say that up to $80 \%$ of the accidents are caused by workers' actions or omissions [9]. It is thus crucial to study both the employees' behavior and personality when assigning tasks. Workers are indeed characterized by the so-called human factors, i.e., individual aspects and organizational, environmental and job factors that modify a worker's behavior in a way that can influence occupational safety and health (OSH) [9]. Human factors affect risk perception, i.e., how one understands characteristics and level of danger in the presence of hazards $[3,4,16,18,19]$. Human factors 
include, e.g., age, past experience and health status, social and cultural aspects, psychological traits, trust in risk management institutions, optimism bias $[7,11]$ and locus of control $[10,11]$. These factors have been investigated in the Sociology and Psychology literature, but the way they affect human behavior when in the presence of risk remains vague.

Risk awareness courses help employees achieve appropriate risk awareness and enforce safety guidelines be observed. Employees regularly undergo risk awareness training with high capital investments for companies. However, fatalities and accidents at work are frequent. Novel techniques were proposed in [12] and [13] to profile workers depending on their sensitivity to risk. This can help provide employees tailored risk awareness courses. Also, the Smart Manufacturing approach, part of the groundbreaking Industry 4.0 paradigm, has the workplace safety improvement as a primary objective. Limited economic resources make safety hard to manage for Small and Medium Enterprises (SMEs), which represent $\sim 99 \%$ of the companies in the EU and employ 65 million people [2]. If human factors were included into OSH procedures, accidents at work could be reduced [9]. To help SMEs achieve a low-cost workplace safety increase, it may be sufficient to reallocate the personnel to tasks analyzing the employees': i) human factors; ii) ability to learn new tasks; iii) behavior when exposed to the hazards of the tasks [14], [15].

This paper presents a multi-objective approach to reallocate the personnel of a company to the tasks in order to improve the workplace safety, while keeping low both the costs and the time to learn the new tasks assigned. The learning time an employee takes to learn a new task is predicted using his/her past jobs where the employee performed that task. Risk-free practical tests help estimate the learning time in the case the worker has never performed the task before.

A neural network-based system [6] calculates every employee's level of caution towards each task, starting from the human factors and behavior when in the presence of the risks of the task. An employee's behavior while performing a task is expressed on the basis of the precautions taken during the task execution.

The multi-objective problem is solved by generating an approximation of the whole Pareto front using the Non-dominated Sorting Bee Colony optimization algorithm (NSBC). The most appropriate Pareto-optimal personnel (re)allocation is selected using the TOPSIS algorithm. Experiments were carried out involving two footwear companies with 11 and 25 employees, respectively.

The paper is organized as follows: Section 2 contains a background on multiobjective optimization; Section 3 describes TOPSIS; Section 4 contains the details on how an employee's level of caution towards a task is computed; Section 5 gives the problem formulation; in Section 6 the experiments are discussed; Section 7 draws the conclusions.

\section{Multi-objective optimization}

Multi-objective optimization (MOO) problems deal with the optimization of multiple objectives, typically conflicting [8]. An MOO problem can be written 
as Minimize $_{\mathbf{x} \in \mathcal{X}} \mathbf{f}(\mathbf{x})=\left[f_{1}(\mathbf{x}), \ldots, f_{k}(\mathbf{x})\right]$, where $\mathcal{X}=\left\{x \in \mathbb{R}: g_{i}(\mathbf{x}) \leq 0 \forall i=\right.$ $\left.1, \ldots, m, h_{j}(\mathbf{x})=0 \forall j=1, \ldots, n\right\}$. The vector function $\mathbf{f}: \mathbb{R}^{p} \rightarrow \mathbb{R}^{k}$ contains the objective functions. In general, any solution does not minimize all the objective functions at the same time. Thus, Pareto dominance and Pareto-optimality are introduced. A solution $\mathbf{x}^{\mathbf{1}}$ dominates $\mathbf{x}^{\mathbf{2}}$ if $f_{i}\left(\mathbf{x}^{\mathbf{1}}\right) \leq f_{i}\left(\mathbf{x}^{\mathbf{2}}\right) \forall i \in\{1, \ldots, k\}$, and $f_{j}\left(\mathbf{x}^{\mathbf{1}}\right)<f_{j}\left(\mathbf{x}^{\mathbf{2}}\right)$ for at least one $j \in\{1, \ldots, k\}$. Pareto-optimal solutions map to the Pareto front, in the objective space.

\subsection{Non-dominated sorting bee colony optimization}

Overview The Non-dominated Sorting Bee Colony (NSBC) algorithm is a popular algorithm inspired by the foraging behavior of bees [17]. NSBC encodes a solution using the position of a food source and its nectar amount (i.e., the fitness of the solution). NSBC divides a bee colony into onlookers, employed bees and scouts. Onlookers stand on a dance area waiting to decide for a quality food source; employed bees are associated with food sources; scouts perform random search. The total number of employed and onlooker bees is equal to the number of candidate solutions.

The NSBC optimization algorithm NSBC initializes a population of $N$ food sources (candidate solutions) of dimension $D$. Food source $\mathbf{X}_{i}(t)=\left(x_{i, 1}, \ldots, x_{i, D}\right)$ of population $P_{t=0}$ is randomly initialized in the range $\left[\mathbf{X}_{\min }, \mathbf{X}_{\max }\right]$, with $\mathbf{X}_{\min }=\left(x_{1}^{\min }, \ldots, x_{D}^{\min }\right)$ and $\mathbf{X}_{\max }=\left(x_{1}^{\max }, \ldots, x_{D}^{\max }\right)$. The $j$-th component of $\mathbf{X}_{i}(t=0)$ is $x_{i, j}(t=0)=x_{j}^{\text {min }}+\mathcal{U}(0,1) \cdot\left(x_{j}^{\text {max }}-x_{j}^{\text {min }}\right)$, where $\mathcal{U}(0,1)$ is a random number uniformly distributed in $[0,1]$. The $k$-th component $f_{k}\left(\mathbf{X}_{i}(t=0)\right)$ if the fitness of every food source $\mathbf{X}_{i}(t=0)$ is computed, for each $i=1, \ldots, N$.

Each employed bee looks for a new food source $\mathbf{X}_{i}^{\prime}(t)=\left(x_{i, 1}, \ldots, x_{i, j}^{\prime}, \ldots, x_{i, D}\right)$ changing the $j$-th component, selected randomly. The new value is:

$$
x_{i, j}^{\prime}(t)=x_{i, j}(t)+\mathcal{U}(-1,1) \cdot\left(x_{i, j}(t)-x_{k, j}(t)\right)
$$

where $k \neq i$ and $\mathcal{U}(-1,1)$ is a random number uniformly distributed in $[-1,1]$. If $\mathbf{X}_{i}(t)$ is dominated by $\mathbf{X}_{i}^{\prime}(t)$, the bee substitutes the previous food source with $\mathbf{X}_{i}^{\prime}(t)$. Otherwise, the bee maintains both solutions in memory. This step iterates for every food source. The population so obtained (size $N \leq N^{\prime} \leq 2 N$ ) is sorted according to non-domination. Non-dominated food sources take rank 1 (first front). These food sources are then neglected to find the second front, etc.

A parent population $P_{t}^{\prime}$ of size $N$ is built for the onlooker bee phase according to the ascending order of the non-domination ranking. Food sources in the last front that can be inserted in $P_{t}$ are sorted in descending order of crowding distance, i.e., the sum of the distances from a food source to its closest food source along each objective. Let $\mathcal{D}_{i}$ be the set of the food sources dominated by $\mathbf{X}_{i}(t)$. Each food source $\mathbf{X}_{i}(t=0)$, where $i=1, \ldots, N$, is associated with a probability to be selected by the onlooker bee, which is equal to $\pi_{i}=\frac{\left|\mathcal{D}_{i}\right|}{N}$, where $|\cdot|$ denotes the cardinality of the set. Onlooker bees evaluate the fitness of each 
food source from all employed bees and select a food source $\mathbf{X}_{i}(t)$ on the basis of probability $\pi_{i}$. Onlooker bees change the position of the food source in their memory in accordance to Eq. (1) and test the fitness of the new food source.

Population $P_{t}^{\prime}\left(\right.$ size $\left.N \leq N^{\prime} \leq 2 N\right)$ is made up by evaluating the Pareto dominance between the neighborhood and the previous food sources. As employed bees do, using the crowding distance non-domination sorting, the non-dominated food sources in $P_{t}^{\prime}$ are found to build population $P_{t+1}$ of size $N$.

Finally, when a food source is not improved throughout a certain number of epochs, it is replaced by a randomly position found by the scouts. NSBC iterates until a stop condition is met.

\section{Technique for Order of Preference by Similarity to Ideal Solution (TOPSIS)}

TOPSIS is an MCDM approach [5]. Considering a decision problem characterized by $n$ alternatives and $m$ criteria, TOPSIS requires an $n \times m$ decision matrix $\mathbf{H}=\left[h_{i j}\right]$, where $i \in\{1, \ldots, n\}$ and $j \in\{1, \ldots, m\}$. The goodness of alternative $i$ w.r.t. criterion $j$ is measured by element $h_{i j}$. In addition, criteria must be prioritized by assigning them weights. Let these weights be contained in a vector $\boldsymbol{\omega}=\left(\omega_{1}, \ldots, \omega_{m}\right)$, with $\sum_{j=1}^{m} \omega_{j}=1$. TOPSIS first calculates the weighted normalized decision matrix $\mathbf{V}=\left[v_{i j}\right]=\omega_{j} h_{i j} / \sqrt{\sum_{i=1}^{n} h_{i j}^{2}}$, then it finds the ideal best $(I B)$ and worst $(I W)$ solutions. The indices in $\Omega_{B}$ and $\Omega_{C}$ indicate benefit and cost criteria, respectively. Let $I B=\left(a_{1}^{+}, \ldots, a_{m}^{+}\right)$and $I W=\left(a_{1}^{-}, \ldots, a_{m}^{-}\right)$, where $a_{j}^{+}=\max _{i} v_{i j}$ for $j \in \Omega_{B}$ or $a_{j}^{+}=\min _{i} v_{i j}$ for $j \in \Omega_{C}$, and $a_{j}^{-}=\min _{i} v_{i j}$ for $j \in \Omega_{B}$ or $a_{j}^{-}=\max _{i} v_{i j}$ for $j \in \Omega_{C}$. TOPSIS measures the Euclidean distance of every single alternative from $I B$, namely, $D_{i}^{+}=\sqrt{\sum_{j=1}^{m}\left(v_{i j}-a_{j}^{+}\right)^{2}}$, and $I W$, i.e., $D_{i}^{-}=\sqrt{\sum_{j=1}^{m}\left(v_{i j}-a_{j}^{-}\right)^{2}}$. TOPSIS eventually measures the relative closeness coefficient of every alternative to $I B$ as $R C L_{i}^{+}=D_{i}^{-} /\left(D_{i}^{+}+D_{i}^{-}\right)$: the higher $R C L_{i}^{+}$the better the alternative. The alternative $k=\arg \max _{i} R C L_{i}^{+}$ is chosen and results to be the best.

\section{Worker's risk perception and caution}

Consider a set of tasks $\mathcal{T}=\left\{t_{1}, \ldots, t_{|\mathcal{T}|}\right\}$ and a set of employees $\mathcal{E}=\left\{e_{1}, \ldots, e_{|\mathcal{E}|}\right\}$. An employee is assumed to be exposed to a set $\mathcal{R}_{i}$ of risks when performing task $t_{i}$. Let the set of risks of the workplace be $\mathcal{R}=\bigcup_{i=1}^{|\mathcal{T}|} \mathcal{R}_{i}$. Each employee innately takes specific precautions when exposed to the risks of a task. Formally, let set $\mathcal{A}_{k}=\left\{a_{k, 1}, \ldots, a_{k,\left|\mathcal{A}_{k}\right|}\right\}$ contain preventive actions, i.e., precautions an employee can take to mitigate a risk $r_{k} \in \mathcal{R}$, where $k \in\{1, \ldots,|\mathcal{R}|\}$. Preventive actions can mitigate a risk, i.e., they decrease the risk occurrence and/or its impact. Depending on this extent, each preventive action is associated with a level of prevention in $\mathcal{L}=\{1, \ldots, L\}$. The more the action makes a risk less likely 
and/or mitigates its impact, the higher the level of prevention. Experts in risk assessment assign the levels of prevention to preventive actions.

Consider a set $\mathcal{H}=\left\{h_{1}, \ldots, h_{|\mathcal{H}|}\right\}$ of human factors (or factors). Each $h_{v}$ takes values in a domain $\mathcal{D}_{v}$. Set $\mathcal{H}$ is made of factors relating the worker's past history and work experience, and the ones relating to the task. The first set is composed of $P$ personal factors. The second contains $T$ task-related factors. The risk perception personal level pers_perc ${ }_{j}$ of employee $e_{j}$ stems from the set $\mathcal{P}_{j}=\bigcup_{v=1}^{P} d_{v, j}$, which contains the values $d_{v, j} \in \mathcal{D}_{v}$ of every personal factor $h_{v}$.

A function $\varphi_{P E R S O N A L}$ such that $\mathcal{P}_{j} \mapsto \varphi_{P E R S O N A L}\left(\mathcal{P}_{j}\right)=$ pers $_{-}$perc $c_{j}$ exists, and the perception level task_perc $c_{i, j}$ of $e_{j}$ for the risks of task $t_{i}$ is established by $\mathcal{T}_{j}=\bigcup_{v=P+1}^{P+T} d_{v, j}$. Here, $d_{v, j}$ are the values of task-related factors $h_{v}$ for employee $e_{j}$. The risk perception personal level pers_perc $c_{j}$ of $w_{j}$ also influences task_perc $c_{i, j}$. Thus, there exists a function $\varphi_{T A S K}$ such that $\left(\mathcal{T}_{j}\right.$, pers_perc $\left._{j}\right) \mapsto \varphi_{T A S K}\left(\mathcal{T}_{j}\right.$, pers_perc $\left._{j}\right)=$ task_perc $_{i, j}$.

For each risk $r_{k}$ and employee $e_{j}$, the caution of $e_{j}$ for $r_{k}$ is measured on the basis of the number of preventive actions per level of prevention that $e_{j}$ performs when exposed to $r_{k}$ : this is the behavior of $e_{j}$ towards $r_{k}$. Let us denote the number of $\bar{\ell}$-level preventive actions $e_{j}$ performs when exposed to $r_{k}$ as $\# \mathcal{A}_{k, \ell=\bar{\ell}, j}$. A function $\rho_{k}$ such that $\left(\# \mathcal{A}_{k, \ell=1, j}, \ldots, \# \mathcal{A}_{k, \ell=L, j}\right) \mapsto \rho_{k}\left(\# \mathcal{A}_{k, \ell=1, j}, \ldots, \# \mathcal{A}_{k, \ell=L, j}\right)$ $=$ risk_caution $_{k, j}$ can thus be configured for each $k=1, \ldots,|\mathcal{R}|$.

For each task $t_{i}$ and employee $e_{j}$, the caution of employee $e_{j}$ when performing task $t_{i}$ therefore depends on risk_caution $_{k, j}, \forall k \in \mathcal{R}_{i}$. For this reason, a group of functions $\tau_{i}$, one for each $i=1, \ldots,|\mathcal{T}|$, such that $\bigcup_{r_{k} \in \mathcal{R}_{i}}$ risk_caution $_{k, j} \mapsto$ $\tau_{i}\left(\bigcup_{r_{k} \in \mathcal{R}_{i}}\right.$ risk_caution $\left._{k, j}\right)=$ task_caution $_{i, j}$, computes the level of caution of employee $e_{j}$ for each task $t_{i}$, given the employee's levels of caution for the risks involved. A tuple $\theta_{j}=\left\{\bigcup_{v=1}^{P+T} d_{v, j}, \bigcup_{k=1}^{|\mathcal{R}|} \bigcup_{\lambda=1}^{L} \# \mathcal{A}_{k, l=\lambda, j}\right\}$ therefore represents the employee $e_{j}$ in the model here given. It is important to point out that $|\mathcal{H}|=P+T$ and $v \in\{1, \ldots, P\}$ refers to personal factors, whereas task-related factors are referred to as $v \in\{P+1, \ldots, P+T\}$.

Given tuple $\theta_{j}$, the levels of risk perception and caution of each employee towards every task are determined in this paper using the neural network-based system whose architecture and training process are described in detail in [6].

\section{Problem formulation}

\subsection{Objectives}

Consider a decision variable $x_{i j} \in\{0,1\}$. Let $x_{i j}=1$ if employee $e_{j}$ is reallocated to task $t_{i}$, and let $x_{i j}=0$ if not, where $i \in\{1, \ldots,|\mathcal{T}|\}$ and $j \in\{1, \ldots,|\mathcal{E}|\}$. The vector $\mathbf{x} \in\{0,1\}^{|\mathcal{T}| \times|\mathcal{E}|}$ is a personnel assignment, and has decision variables $x_{i j}$ as elements, in lexicographic order. The three objectives considered in the optimization approach described in this paper are formalized in the next sections.

Cost Assigning task $t_{i}$ to employee $e_{j}$ results in a cost that depends on the employee's work seniority for task $t_{i}$ and his/her need to be trained to perform 
$t_{i}$. The longer $e_{j}$ has performed $t_{i}$ in life, the lower the cost for the training. The cost $c_{i j}$ of assigning employee $e_{j}$ to task $t_{i}$ includes the cost for the training and what the employer pays for salary and benefits. The overall cost objective function $\operatorname{COST}(\mathbf{x}):\{0,1\}^{|\mathcal{T}| \times|\mathcal{E}|} \rightarrow \mathbb{R}^{+}$to minimize is modeled as:

$$
\operatorname{COST}(\mathbf{x})=\sum_{i=1}^{|\mathcal{T}|} \sum_{j=1}^{|\mathcal{E}|} c_{i j} x_{i j}
$$

Learning time In general, more experienced workers are preferred to be assigned to tasks. Consider the average number of days $A V G \_T I M E_{i}$ typically required to employees to be properly trained for each task $t_{i}$. If an employee has never performed the task before, this number of days is assumed to be required to train the worker. If the employee has a past experience for that task, let $\mathcal{P}_{i, j}$ be the set of the past jobs where employee $e_{j}$ performed task $t_{i}$. The experience of employee $e_{j}$ for task $t_{i}$ is estimated as

$$
\text { experience }_{i, j}=\frac{\left|\mathcal{P}_{i, j}\right|}{\sum_{u \in \mathcal{P}_{i, j}} \text { duration }_{u}^{-1}},
$$

where duration du $_{u}$ is the duration (in days) of past job $u$. The harmonic mean is used in Eq. (3) for being able to mitigate (intensify) the impact of large (small) outliers. The time $T_{i, j}$ an experienced employee $e_{j}$ takes to be trained for $t_{i}$ is estimated as:

$$
T_{i, j}=\left\{\begin{array}{cc}
A V G_{-} T I M E_{i} & \text { if } \\
a_{i, j} \cdot A V G_{-} T I M E_{i} & \text { oxperience } \\
i, j & \geq k \cdot A V G_{-} T I M E
\end{array}\right.
$$

where the parameter $k>0$ is set up by experts in the field, and $a_{i, j}>0$ results from risk-free practical tests where experts in the field evaluate how skilled employee $e_{j}$ is in executing task $t_{i}$. The overall learning time is estimated with the mean to variance ratio

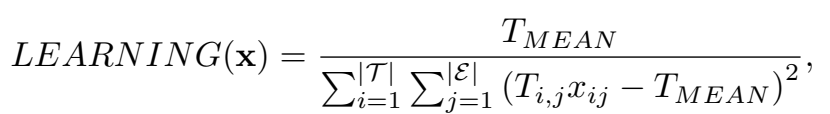

where $T_{M E A N}$ is the average learning time for the tasks assigned defined as: $T_{M E A N}=\frac{1}{|\mathcal{T}|} \sum_{i=1}^{|\mathcal{T}|} \sum_{j=1}^{|\mathcal{E}|} T_{i, j} x_{i j}$

Caution Let us define the average level of caution for the tasks assigned as: $C_{M E A N}=\frac{1}{|\mathcal{T}|} \sum_{i=1}^{|\mathcal{T}|} \sum_{j=1}^{|\mathcal{E}|}$ task_caution $_{i, j} x_{i j}$. The overall level of caution for the tasks assigned $C A U T I O N(\mathbf{x}):\{0,1\}^{|\mathcal{T}| \times|\mathcal{E}|} \rightarrow \mathbb{R}^{+}$to maximize, is defined here as the mean to variance ratio of the level of caution of each employee towards the task assigned:

$$
C \operatorname{AUTION}(\mathbf{x})=\frac{C_{M E A N}}{\sum_{i=1}^{|\mathcal{T}|} \sum_{j=1}^{|\mathcal{E}|}\left(\text { caution }_{i, j} x_{i j}-C_{M E A N}\right)^{2}} .
$$




\subsection{Problem formulation}

Consider a set of tasks $\mathcal{T}$ and a set of employees $\mathcal{E}$ where $|\mathcal{T}|=|\mathcal{E}|$. Each task must be (re)assigned to one worker and vice versa. The optimization problem is:

$$
\underset{\mathbf{x}}{\operatorname{Minimize}} \mathbf{f}(\mathbf{x})=[\operatorname{COST}(\mathbf{x}), \operatorname{LEARNING}(\mathbf{x}),-\operatorname{CAUTION}(\mathbf{x})]
$$

subject to:

$$
\begin{gathered}
\sum_{i=1}^{|\mathcal{T}|} x_{i j}=1, \quad \forall j=1, \ldots,|\mathcal{E}| \\
\sum_{j=1}^{|\mathcal{E}|} x_{i j}=1, \quad \forall i=1, \ldots,|\mathcal{T}| \\
x_{i j} \in\{0,1\}, \quad \forall i=1, \ldots,|\mathcal{T}|, \forall j=1, \ldots,|\mathcal{E}| .
\end{gathered}
$$

Equation (6a) is the objective function $\mathbf{f}(\mathbf{x}):\{0,1\}^{|\mathcal{T}| \times|\mathcal{E}|} \rightarrow \mathbb{R}_{+}^{2} \times \mathbb{R}_{-}$whose components are the overall cost, the average learning time, the overall level of caution (inverted in sign) towards the tasks assigned of assignment (i.e., personnel reallocation) $\mathbf{x} \in\{0,1\}^{|\mathcal{T}| \times|\mathcal{E}|}$. Constraints (6b) force each worker be reallocated to one task. Constraints (6c) let instead each task of the factory be (re)assigned to one worker. Equations (6d) express the integer constraint.

\section{Experiments and Discussion}

The proposed approach was applied to two scenarios ("Scenario A" and "Scenario B") based on two real-world case studies related to two footwear companies. The optimization approach was implemented in MATLAB.

\subsection{Dataset}

A website was implemented to collect information about the employees: the values of their human factors and behavior. The employees were required to fill out a questionnaire through the website. Data were collected in compliance with the privacy laws. For each employee $e_{j}$, the questionnaire collects:

- the values of the human factors in order to compute task_perc $_{i, j}$;

- data relating the past jobs to estimate the learning times;

- data relating the behavior towards each risk $r_{k}$ of every task $t_{i}$ on the basis of the preventive actions the employee chooses from a predefined set of actions.

The actions chosen let task_caution $_{i, j}$ for each task $t_{i}$ be computed.

The dataset consists of 36 interviews: 11 interviews relate to the first company, the other 25 interviews relate to the second company. Due to privacy laws and ethical issues, the dataset cannot be made public domain at the moment. 


\subsection{Setup of the parameters}

The system was implemented in MATLAB and was tested on a workstation equipped with a $3.4 \mathrm{GHz} \mathrm{CPU}$ with $8 \mathrm{~GB}$ of RAM. By means of a trial and error approach, the NSBC algorithm was run for 500 generations and the population size was set to 100 . To find this configuration, a total of 30 trials were run using different values for population size and number of generations. These values were determined on the basis of heuristic considerations on the problem.

\subsection{Optimization results}

Personnel assignment strategy in the involved companies In SMEs, managers determine how suitable an employee is to a task on the basis of experience: the higher the experience the more suitable is the employee. As many tasks of a footwear industry are handmade and require using dangerous machines, workers are continuously exposed to serious risks including crushing injuries, burns and amputation. However, risk management is typically carried out by SMEs by assigning more dangerous tasks to more experienced employees mainly for being unprepared to make capital investments. This is tremendously dangerous because more experienced workers typically have higher locus of control and this can decrease the risk awareness [10].

The shoe making process Making a shoe is a complex process, with many handmade operations. The process starts with cutting pieces of leather using cutting machines and knives to prepare some of the parts of a shoe, i.e., uppers, linings, reinforcements and insoles. Die cutters are used to prepare other parts, such as welts, vamps, soles, heels. The thickness of the leather is made uniform using milling cutters while preparing the upper. Ornaments are then applied to the shoe. By sewing all the parts above, the upper is assembled: this phase is called stitching. A pounding phase lets possible folds of the leather be smoothed. The upper is then mounted on the last (i.e., a sculpture of the shoe) using a lasting machine, and is finally joined with the insole. The sole is applied by using sanding machines, through glueing, sewing or welts. Die casting or nails are used to fix the heel. Heels are typically coated with leather or wrapped with a material similar to the one the upper is made of. A press fixes the upper to the block made of sole and heel, in the case of rubber soles. The bottom of the shoe is finished by: i) sanding heel and sole by using rotating machines; ii) waxing and coloring the sole contour with rotating tools; iii) polishing sole and heel.

The process ends with embellishment steps that include cleaning the upper with solvents/brushes, waxing-up the sole, polishing and starching. The shoes are eventually put in pairs into shoeboxes to be stored into the warehouse.

Proposed strategy for personnel reallocation The proposed approach for personnel reallocation started asking the management to prioritize the objectives. Preventive actions were then classified into three prevention levels: low, 
medium, high. Each employee's data about human factors and behavior were collected by means of the website described in Section 6.1. Each employee's task_perc and risk_caution toward each risk were computed using such data. The neural system referred to in Section 4 computed each employee's levels of task_caution and task_perc towards every task. The Pareto front was approximated by means of NSBC considering a maximum number of iterations equal to 1000. The best solution selected by TOPSIS is in Table 1, for both scenarios.

Discussion of scenario A In this scenario the company has 11 employees. The company aims to improve the workplace safety with low increase in cost. The weights of the objectives are $(0.35,0.2,0.45)$, in the order they appear in Eq. (6a). Cost and caution are thus the most important objectives. The left-hand side of Fig. 1 shows the Pareto front obtained by means of the NSBC algorithm.
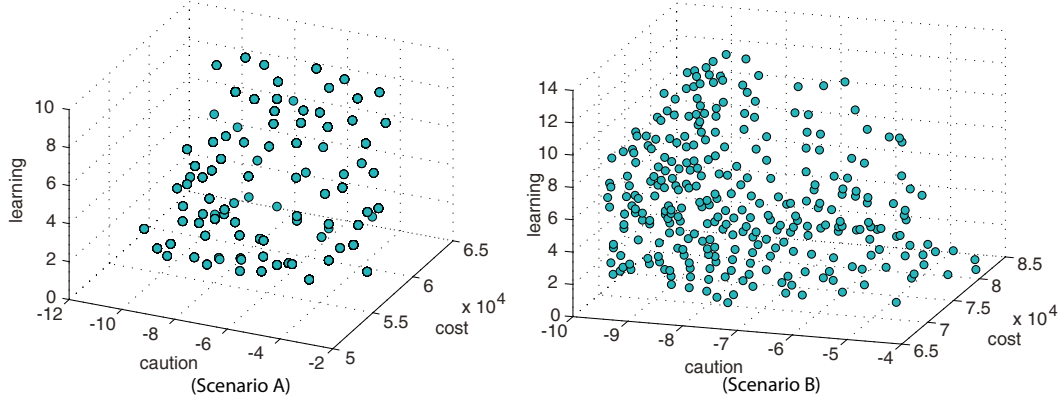

Fig. 1. Pareto front obtained for Scenario A (left) and Scenario B (right).

The current assignment has a cost of $30,180 €$ and shows an overall level of caution of -5.15 , as summarized in Table 1 . Learning is equal to zero because all employees can perform their task correctly.

The proposed solution reassigns 5 tasks (highlighted with colored cells in Table 1) and guarantees $\sim 120 \%$ improvement in the overall level of caution at the expense of a $\sim 18 \%$ increase in cost. Cost is here paramount for the involved company and a percentage like this may seem unreasonable. Anyway, that is not what it seems like because the increase in cost is just temporary, as it is due to the cost of training the reallocated workers.

Data on the employees' behavior w.r.t. every task cannot be reported. Consider that each task of a footwear company exposes an employee to five risks on average. Each risk can in turn be prevented by four actions per level of prevention, on average. Also, three levels of prevention (low, medium and high) are considered. This causes an explosion of the number of preventive actions per employee to report. The discussion is thus based on intuitive considerations.

The proposed approach, if implemented, would guarantee that $\sim 75 \%$ of the employees would deal with the task assigned with high-level preventive actions 
only. The remaining percentage would have behaviors between a poor level of caution (3 low-level actions) and a good level of caution (2 medium-level actions and 2 high-level actions).

It is important to note that the most unsafe behavior (i.e., just 1 low-level action) stems from workers who stamp the insoles or make supervision. As can be understood, these tasks are almost risk-free. Workers may just experience muscle tightness and eyestrain.

Instead, in the current assignment, 2 safety-critical tasks are performed by employees with behavior characterized by 1 low-level preventive action. These tasks involve diecutters and pounding machines, which can be a serious threat for the health if used unsafely. For instance, employees may experience grazes, crushing injuries to the hands and amputation. The employees currently assigned to these tasks are highly likely to get hurt due to their poor level of caution.

Assigning safety-critical tasks to people with a higher number of years of experience is tremendously wrong from the point of view of safety. These workers, of course, perform the task better than others, but the management is highly wrong if neglects their behavior when in the presence of risk.

In the current assignment, safety-critical tasks are assigned to employees with, on average, 15 years of experience for the task. However, their level of caution is poor. As one can imagine, people become familiar with something that is performed every day in life. And this causes a decrease in risk awareness. Accordingly, the employees that the management assigned to safety-critical tasks have become familiar with the task, and they may have inadequate awareness of the risks they take when performing the task.

Regarding the learning time, the proposed reallocation of personnel can guarantee a fast training, $\sim 3$ days, on average, as can be seen from Table 1 .

Table 1. Current and proposed personnel assignment for Scenarios A and B.

\begin{tabular}{|c|c|c|c|c|c|c|c|c|c|c|c|c|c|c|c|c|c|c|c|c|c|c|c|c|c|}
\hline & \multicolumn{24}{|c|}{ TASKS } \\
\hline & & 12 & 2 & 3 & 4 & 5 & 6 & \begin{tabular}{l|l|}
7 & 8
\end{tabular} & 9 & 10 & & & 13 & & & & & 18 & & 20 & $\left.21\right|_{2}$ & 22 & & \begin{tabular}{l|l}
24 & 2
\end{tabular} & \\
\hline & Current & 5 & 2 & 10 & 3 & 7 & 4 & 116 & 1 & 9 & 8 & - & - & - & - & - & - & - & - & - & - & - & - & -1. & - \\
\hline & Proposed & 5 & 9 & 11 & 3 & 2 & 4 & 106 & 1 & 7 & 8 & - & - & - & - & - & - & - & - & - & - & - & - & -7 & - \\
\hline & Current & 23 & & 8 & 21 . & & 6 & \begin{tabular}{l|l|}
25 & 9 \\
\end{tabular} & 11 & 19 & 16 & 5 & 24 & 7 & 18 & 14 & 1 & 4 & & 15 & 13 & 2 & & 122 & 22 \\
\hline & Proposed & 23 & & 10 & 21 & & 22 & \begin{tabular}{|l|l|}
25 & 9 \\
\end{tabular} & 7 & 19 & 16 & 5 & 2 & & 18 & 14 & 20 & 4 & 8 & 15 & 13 & 24 & 11 & 12 & 6 \\
\hline
\end{tabular}

Discussion of scenario B In this scenario, the weights of the objectives are $(0.4,0.1,0.5)$ The management wants thus to reallocate the personnel to improve the safety keeping low the costs.

The current assignment of personnel to tasks is characterized by an overall caution equal to -4.78 (see Table 1 ). By reallocating the personnel as suggested 
Table 2. Values of the objectives for Scenarios A and B.

\begin{tabular}{|c|c|c|c|c|}
\cline { 3 - 5 } \multicolumn{2}{c|}{} & COST $[€]$ & LEARNING & -CAUTION \\
\hline \multirow{2}{*}{ A } & Current & $30,180.00$ & 0 & -5.15 \\
\cline { 2 - 5 } & Proposed & $35,720.00$ & 3.22 & -11.41 \\
\hline \multirow{2}{*}{ B } & Current & $68,995.00$ & 0 & -4.78 \\
\cline { 2 - 5 } & Proposed & $76,150.00$ & 5.38 & -9.94 \\
\hline
\end{tabular}

by the proposed approach, the overall level of safety is more than doubled at the expense of a temporary increase in cost of $\sim 10 \%$. The Pareto front is in Fig. 1. The behaviors that highly impact on safety are discussed in the following.

As an example, it is fundamental to observe that the safest behaviors, i.e., the ones made of high-level preventive actions only, pass from 5 (in the current personnel assignment) to 9 thanks to the proposed reallocation of personnel to tasks. Also, with the proposed solution no employee behaves showing low-level preventive actions only. In the current assignment, there are instead 6 employees characterized by behaviors like this, the two most hazardous of them involve a task where employees may experience crushing injuries while using diecutters and severe excoriations, respectively. This is another SME where the management chooses more experienced employees for the most dangerous tasks.

Finally, the time required by the employees to learn the new tasks assigned is estimated to be a bit longer than 5 days (see Table 1 ). This is a short amount of time if one thinks that 10 tasks (colored cells in Table 1 ) are reassigned. The time required to learn the new tasks assigned is thus compliant with the importance assigned by the management to the corresponding objective.

\section{Conclusion}

This paper has presented a multi-objective optimization approach for personnel reallocation aimed at improving the workplace safety in SMEs, while maintaining low the costs and the time required to train employees to perform the new tasks. Each employee's level of caution for every task is determined by a neural network-based system on the basis of some human factors and the precautions the employee takes when performing a task. The NSBC algorithm and TOPSIS are used to find a Pareto-optimal personnel reallocation.

The approach was tested in two footwear companies. A high improvement of the level of caution was obtained in both scenarios with a low and temporary increase in cost. Risks thus become less harmful and less likely as tasks are assigned to more appropriate employees w.r.t. their level of caution while performing the tasks. This is key for SMEs, a widespread reality in the EU.

\section{References}

1. http://www.ilo.org. International Labour Organization Statistics Database (ILOSTAT) 
2. Bandini, S., Manzoni, S., Sartori, F.: Case-based reasoning to support work and learning in small and medium enterprises. In: 21st IEEE Int. Conf. on Tools with Artificial Intelligence. pp. 253-260 (2009)

3. Bouyer, M., Bagdassarian, S., Chaabanne, S., Mullet, E.: Personality correlates of risk perception. Risk Analysis 21(3), 457-465 (2001)

4. Chauvin, B., Hermand, D., Mullet, E.: Risk perception and personality facets. Risk Analysis 27(1), 171-185 (2007)

5. Ching-Lai Hwang, K.Y.: Multiple attribute decision making. Springer-Verlag (1981)

6. Cococcioni, M., Lazzerini, B., Pistolesi, F.: A semi-supervised learning-aided evolutionary approach to occupational safety improvement. In: IEEE Congress on Evolutionary Computation (2016)

7. Costa-Font, J., Mossialos, E., Rudisill, C.: Optimism and the perception of new risks. Journal of risk research 12(1), 27-41 (2009)

8. Deb, K., Kalyanmoy, D.: Multi-Objective Optimization Using Evolutionary Algorithms. John Wiley \& Sons, Inc. (2001)

9. Health, Safety Executive: Reducing error and influencing behaviour. HSE Books (1999)

10. Horswill, M.S., McKenna, F.P.: The effect of perceived control on risk taking. Journal of Applied Social Psychology 29(2), 377-391 (1999)

11. Klein, C.T.F., Helweg-Larsen, M.: Perceived control and the optimistic bias: a meta-analytic review. Psychology and health 17(4), 437-446 (2002)

12. Lazzerini, B., Pistolesi, F.: Profiling risk sensibility through association rules. Expert Systems with Applications 40(5), 1484-1490 (2013)

13. Lazzerini, B., Pistolesi, F.: Classifying workers into risk sensibility profiles: a neural network approach. In: 8th European Modelling Symposium. pp. 33-38 (2014)

14. Lazzerini, B., Pistolesi, F.: An integrated optimization system for safe job assignment based on human factors and behavior. IEEE Systems Journal (2017), 10.1109/JSYST . 2016.2646843

15. Lazzerini, B., Pistolesi, F.: Multiobjective personnel assignment exploiting workers' sensitivity to risk. IEEE Transactions on Systems, Man, and Cybernetics: Systems (2017), 10.1109/TSMC. 2017.2665349

16. Peters, E., Slovic, P.: The role of affect and worldviews as orienting dispositions in the perception and acceptance of nuclear power. Journal of applied social psychology 26, 1427-1428 (1996)

17. Rakshit, P., Sadhu, A.K., Bhattacharjee, P., Konar, A., Janarthanan, R.: MultiRobot Box-Pushing Using Non-dominated Sorting Bee Colony Optimization Algorithm, pp. 601-609. Springer Berlin Heidelberg (2011)

18. Sjöberg, L.: Factors in risk perception. Risk Analysis 20(1) (2000)

19. Slovic, P.: Perception of risk. Science 236(4799), 280-285 (1987) 